\title{
Produção de artigos em Psicodrama no Brasil: revisão sistemática (1996- 2014)
}

\author{
Production of Brazilian Psychodrama articles: systematic review (1996-2014)
}

Producción de artículos en Psicodrama en Brasil: revisión sistemática (19962014)

\author{
Paulo Coelho Castelo Branco \\ Universidade Federal da Bahia - e-mail: pauloccbranco@gmail.com \\ Heitor Blesa Farias \\ Universidade Federal da Bahia - e-mail: h_heitor@hotmail.com \\ Cândida de Oliveira Carpes \\ Universidade Federal da Bahia - e-mail: cândida.carpes@hotmail.com \\ Laryssa Soares Leite \\ Universidade Federal da Bahia - e-mail: laryssaasoares245@gmail.com
}

\section{Resumo}

Objetiva-se analisar as produções de Psicodrama em periódicos brasileiros. O método utilizado foi a revisão sistemática de literatura sobre 98 artigos, publicados em 1996-2014, organizados nas seguintes categorias: ano, tipo de produção, área de discussão, periódico e filiação institucional. Os resultados demonstram: intensificação de produções em 2011-2014, predominância de artigos teóricos, hegemonia de discussões clínicas, concentração de publicações em um periódico e ampla difusão de filiações nacionais e estrangeiras relacionadas aos autores que produziram artigos sobre o Psicodrama. Conclui-se que o Psicodrama tem circulado em meios de divulgação acadêmica no Brasil, ampliando a herança de Jacob Moreno concernente à interface entre conhecimento prático e conhecimento científico.

Palavras-chave: Bases de Dados. Psicodrama. Revisão de Literatura.

\section{Abstract}

The objective is to analyse articles on Psychodrama in Brazilian periodicals. The method used was the systematic review of literature for 98 articles published from 1996-2014, organized in the following categories: year; type of article; area of discussion; periodical; institutional 
affiliation. The results show: increased production from 2011-2014; predominance of theoretical articles; predominance of clinical discussions; concentration of publications in a periodical; wide dissemination of national and international affiliations related to authors who have produced articles on Psychodrama. We conclude that Psychodrama has been presented in academic circles of dissemination in Brazil, expanding the legacy of Jacob Moreno concerning the interface between practical knowledge and scientific knowledge.

Keywords: Database. Psychodrama. Literature Review.

\section{Resumen}

El objetivo es analizar la producción de artículos de Psicodrama en periódicos brasileños. El método utilizado fue la revisión sistemática de la literatura en 98 artículos publicados en 1996-2014, organizado en las siguientes categorías: años; tipo de producción; área de discusión; periódico; afiliación institucional. Los resultados muestran: aumento de la producción en 2011-2014; predominio de artículos teóricos; hegemonía de discusiones clínicas; concentración de publicaciones en un único periódico; amplia difusión de las afiliaciones nacionales e internacionales relacionados con los autores que han producido artículos de psicodrama. Se concluye que el Psicodrama ha circulado en los medios académicos de difusión en Brasil, ampliando la herencia de Jacob Moreno relativo a la interfaz entre el conocimiento práctico y el conocimiento científico.

Palabras-clave: Bases de Datos. Psicodrama. Revisión de Literatura.

\section{INTRODUÇÃO}

O Psicodrama é uma abordagem que circula entre os campos da Psicologia, da Medicina e da Psicoterapia. Seu fundador, Jacob Moreno, foi influente/pioneiro na criação de uma proposta de intervenção grupal e pesquisa relacional que faz interface com esses campos. Sabe-se que Moreno se expressou também como figura atuante na criação de várias revistas, associações e grupos/programas de formação que divulgaram suas ideias e outras afins (ROJAS-BERMÚDEZ, 1977; MARINEAU, 1992). Ele deixou, pois, uma herança que articula o conhecimento prático obtido pelo Psicodrama com o conhecimento disseminado em periódicos (MARINEAU, 2013).

No que concerne à manifestação dessa herança no Brasil, destacamos o apontamento importante de Gulassa e Kaufman (2014) sobre a necessidade de mais diálogos do Psicodrama e seus profissionais com o universo científico. Dessa forma, inspiramo-nos nesse apontamento que indica os artigos científicos publicados em periódicos acadêmicos como fonte legítima de conhecimento e formação de opinião. Os autores citados, em consonância com o trabalho de Marineau (2013), explicitam a importância que Moreno deu a esse viés de divulgação e propagação do conhecimento psicodramático.

Com efeito, objetivamos analisar o perfil das produções de artigos publicados sobre o Psicodrama no Brasil. Argumentamos, pois, que essa proposta é útil para entender como a herança de Moreno circula nos espaços acadêmicos de publicação sobre o Psicodrama.

Em relação à existência de outra investigação semelhante a nossa, no Brasil, destacamos a recente pesquisa de Liberali e Grosseman (2015) que apresentam uma revisão sistemática de literatura sobre o uso do Psicodrama na Medicina brasileira, de 2003 até 2013. 
Embora tenha o foco temático mais restrito do que o objetivo de nossa pesquisa, os mencionados autores demonstram que existe uma produção escassa de artigos (total de sete) que divulgam as experiências psicodramáticas nas escolas médicas brasileiras.

Destarte, procedemos da seguinte lógica para cumprir com o objetivo proposto: inicialmente, indicamos o método utilizado para compilar e analisar os artigos de Psicodrama publicados no Brasil; em seguida, apresentamos os resultados dessa coleta e suas respectivas análises que perfazem o perfil da circulação do Psicodrama em periódicos brasileiros.

\section{MÉTODO}

Empregamos a revisão sistemática de literatura como método para coletar e analisar as produções de Psicodrama em periódicos brasileiros. O propósito desse método é elaborar um processo de organização e avaliação de variados artigos sobre um determinado assunto que estão dispersos em diversos periódicos. Seguindo a orientação metodológica postulada por Akobeng (2005), estabelecemos as etapas-guias que serão descritas a seguir.

Inicialmente, delimitamos a seguinte questão de pesquisa: qual é o perfil de produção de conhecimento sobre o Psicodrama que circula em periódicos brasileiros? Em seguida, escolhemos duas bases de dados para realizar nossa investigação, o Scientific Electronic Library Online (SciELO) e o Portal de Periódicos Eletrônicos em Psicologia (PePSIC) ambos escolhidos em razão da amplitude de indexadores, periódicos e popularidade.

No índice de assuntos do SciELO, elegemos as seguintes palavras-chave para a busca de artigos: Psicodrama, Psicodrama Infantil, Psicodrama Pedagógico, Psicodrama Psicanalítico Individual, Psicodrama Psicoanalítico Individual, Psicodramática. No mesmo índice do PePSIC, investigamos os seguintes termos: Psicodrama, Psicodrama Brasileiro, Psicodrama com Crianças, Psicodrama com Crianças e Adolescentes, Psicodrama Organizacional, Psicodrama por Telefone, Psicodrama Público, Psicodrama Socioeducacional, Psicodramática, Psicodramáticas, Psicodramático, Psicodramáticos, Psicodramatista e Psicodramatistas. Com os descritores mencionados, buscamos e armazenamos uma amostra representativa de artigos sobre o Psicodrama publicados no Brasil. Destacamos que essa etapa foi realizada sem o cruzamento dos descritores, havendo busca somente pelas informações que cada termo individualmente gerou no sistema de dados. Os opúsculos foram lidos conforme os títulos, os resumos e as palavras-chave.

Ressaltamos que os artigos foram selecionados de acordo com os seguintes critérios de inclusão: ser publicado em periódicos nacionais e apresentar como conteúdo qualquer discussão teórica, prática ou de pesquisa relacionada ao Psicodrama. Artigos repetidos foram descartados e registrados somente uma vez. Os artigos publicados em 2015 foram descartados, pois era o ano corrente, e havia muitos periódicos que ainda não tinham publicado todos os seus volumes. Logo, a delimitação temporal da coleta foi de 1996, ano da produção mais antiga minutada, até 2014, quando foi possível compilar integralmente todas as produções lançadas nas bases dados.

Em seguida, categorizamos os artigos selecionados segundo: ano, filiação(ões) institucional(is) do(s) autor(es), periódico, tipo (teórico, empírico ou relato de experiência) e área do conhecimento. Essa etapa do processo foi realizada por quatro juízes independentes, a fim de diminuir os vieses no processo de busca/categorização. Em casos de dúvidas na tabulação final, fizemos leituras integrais do artigo e ponderações conjuntas para gerar um consenso. O período de coleta e tabulação ocorreu nos meses de setembro a novembro de 2015. Conforme os postulados atuais do método (AKOBENG, 2005), expressamos em seguida, na Figura 1, o fluxograma que descreveu o plano de seleção/extração de artigos que resultou na amostra bibliográfica final. 


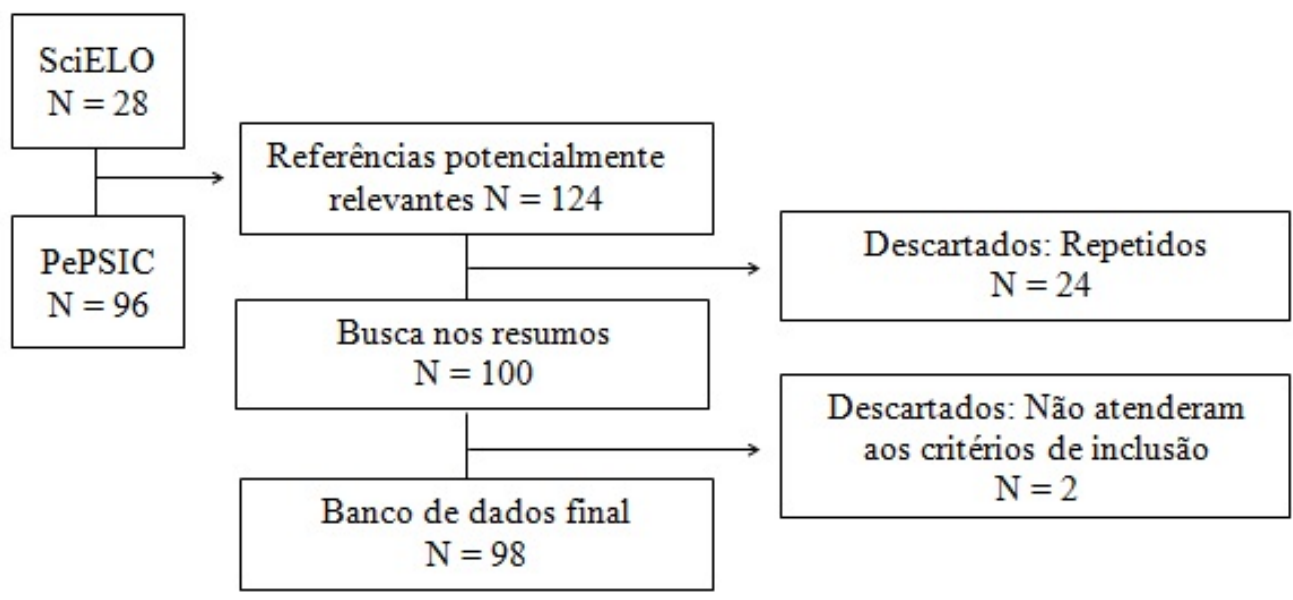

Figura 1. Estratégia de Busca

O PePSIC apresentou o maior número de artigos relacionados ao Psicodrama, possivelmente por sua orientação ser exclusivamente para armazenar/divulgar periódicos especializados em Psicologia. O banco final de artigos $(\mathrm{N}=98)$ compilados e organizados, de maneira pormenorizada, serviu de fonte para diversas análises sobre o perfil da produção de artigos sobre o Psicodrama no cenário brasileiro. Apresentamos, no tópico seguinte, uma síntese dos resultados e das discussões sucedidas pela aplicação do método.

\section{RESULTADOS E DISCUSSÃO}

Os resultados foram processados, inicialmente, conforme as descrições dos dados, dispostos em seguida na Tabela 1, relacionados à distribuição temporal de artigos publicados sobre o Psicodrama no ano de 1996 até 2014.

Os dados expressos na Tabela 1 permitem verificar que o biênio 2012-2013 foi o mais produtivo em relação aos artigos publicados sobre o Psicodrama. Notamos que de 1996 a 2003 existem períodos oscilatórios de anos com poucas publicações e sem produções. Contudo, de 2004 a 2014, o Psicodrama encontrou um momento de estabilidade em termos de publicações contínuas. Observamos que os anos de 2011 a 2014 representam o ápice das produções psicodramáticas em periódicos brasileiros. Esses anos concentram mais da metade da produção nacional catalogada $(n=62 ; 63,25 \%)$, denotando que o Psicodrama começa a intensificar seu diálogo com periódicos acadêmicos.

Em relação à distribuição dos tipos de artigos, observamos uma predominância de publicações de cunho teórico $(\mathrm{n}=52 ; 53,06 \%)$, seguido de produções empíricas $(\mathrm{n}=31$; $31,63 \%)$ e relatos de experiência $(n=15 ; 15,31 \%)$. Ressaltamos que fizemos essa distribuição segundo as categorias de submissão de artigos em periódicos brasileiros. Os aspectos pesquisados expressam produções teóricas preocupadas em elaborar aportes conceituais do Psicodrama, servindo de suporte para (re)leituras do legado de Moreno. A despeito da predominância de artigos teóricos, chama-nos atenção a considerável produção de artigos empíricos que demonstram preocupação em realizar pesquisas que utilizem os referenciais de Moreno ou investiguem os aportes do Psicodrama em seus variados contextos de atuação. 


\begin{tabular}{c|c|c}
\hline Ano & Frequência & \% \\
\hline 1996 & 1 & 1,02 \\
\hline 1997 & - & - \\
\hline 1998 & - & - \\
\hline 1999 & 1 & 1,02 \\
\hline 2000 & 1 & 1,02 \\
\hline 2001 & 2 & 2,04 \\
\hline 2002 & 1 & 1,02 \\
\hline 2003 & - & - \\
\hline 2004 & 3 & 3,06 \\
\hline 2005 & 4 & 4,08 \\
\hline 2006 & 4 & 4,08 \\
\hline 2007 & 4 & 4,08 \\
\hline 2008 & 5 & 5,10 \\
\hline 2009 & 6 & 6,12 \\
\hline 2011 & 4 & 4,08 \\
\hline 2012 & 15 & 15,31 \\
\hline 2013 & 16 & 16,33 \\
\hline 2014 & 16 & 16,33 \\
\hline Total & 15 & 15,31 \\
\hline
\end{tabular}

Tabela 1. Distribuição Temporal de Publicações

Outra análise realizada concerne ao levantamento das áreas de discussões minutadas com base nos artigos coletados, cujos os resultados são apresentados a seguir na Tabela 2.

\begin{tabular}{c|c|c}
\hline Área & Frequência & $\mathbf{\%}$ \\
\hline Clínica & 33 & 33,67 \\
\hline Educação & 16 & 16,33 \\
\hline Social & 16 & 16,33 \\
\hline Organizacional & 7 & 7,15 \\
\hline Saúde & 6 & 6,12 \\
\hline Arte & 6 & 6,12 \\
\hline História/Epistemologia & 6 & 6,12 \\
\hline Desenvolvimento & 5 & 5,10 \\
\hline Avaliação Psicológica & 1 & 1,02 \\
\hline Linguística & 1 & 1,02 \\
\hline Esporte & 1 & 1,02 \\
\hline Total & 98 & 100,00
\end{tabular}

\section{Tabela 2. Distribuição das Áreas de Discussão}

Com efeito, a área clínica apresentou em sua discussão maior expressão de artigos psicodramáticos $(33,67 \%)$, seguido das áreas educacionais $(16,33 \%)$ e sociais $(16,33 \%)$. A hegemonia da clínica, possivelmente, remete-se à tradição do Psicodrama que, em seu cerne, elabora uma proposta ampla de ideias com implicações psicoterapêuticas que podem ser aplicadas em diversas situações. Podemos observar que as produções analisadas exprimem 
uma heterogeneidade de discussões em várias áreas do conhecimento, significando que o saber psicodramático publicado no Brasil é versátil em suas interlocuções.

No que reporta à distribuição de artigos pelos meios acadêmicos que os publicaram, segundo a Tabela 3, constatamos uma diversidade de periódicos.

\begin{tabular}{c|c|c}
\hline Periódicos & Frequência & \% \\
\hline $\begin{array}{c}\text { Revista Brasileira de } \\
\text { Psicodrama }\end{array}$ & 63 & 64,30 \\
\hline $\begin{array}{c}\text { Estudos de Psicologia } \\
\text { (Campinas) }\end{array}$ & 4 & 4,08 \\
\hline $\begin{array}{c}\text { Psicologia para América } \\
\text { Latina }\end{array}$ & 4 & 4,08 \\
\hline $\begin{array}{c}\text { Interface- Comunicação, } \\
\text { Saúde, Educação }\end{array}$ & 3 & 3,06 \\
\hline $\begin{array}{c}\text { Psicologia: Ciência e } \\
\text { Profissão }\end{array}$ & 3 & 3,06 \\
\hline Psicologia em Estudo & 3 & 3,06 \\
\hline Revista da SPAGESP & 2 & 2,04 \\
\hline Paidéia & 2 & 2,04 \\
\hline $\begin{array}{c}\text { Revista Brasileira de } \\
\text { Psiquiatria }\end{array}$ & 2 & 2,04 \\
\hline $\begin{array}{c}\text { Revista Brasileira de } \\
\text { Educação Médica }\end{array}$ & 8 & 2,04 \\
\hline $\begin{array}{c}\text { Revista Latinoamericana de } \\
\text { Psicopatologia Fundamental }\end{array}$ & 98 & 8,16 \\
\hline Outros Periódicos & 2 & 100,00 \\
\hline Total &
\end{tabular}

Tabela 3. Distribuição de Periódicos

Antes de analisar essa Tabela, salientamos que o termo "Outros Periódicos" foi criado para congregar aquelas revistas que somente publicaram um artigo psicodramático. Conquanto haja heterogeneidade de produções em vários periódicos, notoriamente, verifica-se uma concentração de publicações na Revista Brasileira de Psicodrama (64,30\%), que apresenta em seu foco e escopo editorial uma orientação direcionada para publicar artigos em Psicodrama e seus afluentes sociodinâmicos, sociométricos, sociátricos etc. De um ponto de vista interno à construção e à consolidação do saber psicodramático no Brasil, é importante encontrar um periódico especializado e locado no sistema Qualis-CAPES, que seja referência na divulgação de produções do Psicodrama no Brasil. Por outro lado, na perspectiva externa e analítica da propagação desse conhecimento, essa concentração expressa circularidade restrita a somente um meio de publicação acadêmica. Consideramos a circulação de artigos em periódicos de orientações editoriais mais gerais, ou focadas em outros temas, um modo profícuo de produção, difusão, consolidação e divulgação do conhecimento psicodramático para outros públicos. Eis um desafio para o cenário nacional empenhado e gerar novas produções em Psicodrama.

Por fim, em última análise, focamos as filiações institucionais dos 199 autores registrados nos 98 artigos computados. Em razão do extenso número de filiações catalogadas, é inviável reproduzir a tabela gerada no espaço deste artigo. Contudo, indicamos suscintamente alguns exames desse resultado. Há 62 filiações registradas e 18 autores que não 
notificaram suas filiações. Das instituições registradas concentram maior autoria de artigos em Psicodrama, em um ranque de cinco: Universidade de São Paulo $(n=14)$, Sociedade de Psicodrama de São Paulo $(\mathrm{n}=13)$, Universidade de Brasília $(\mathrm{n}=11)$, Universidade Estadual Paulista $(n=11)$ e Universidade Estadual do Ceará $(n=9)$. Em relação aos autores com filiações institucionais estrangeiras, encontramos 16 ocorrências que representam $8,02 \%$ do total das vinculações obtidas. A Universidade de Pádua (Itália) registra cinco filiações, seguida da Universidade de Aix-Marseille (França) que minuta três. Existe, pois, um circuito de autores com vinculações estrangeiras que publicam artigos de Psicodrama em periódicos brasileiros.

Em suma, podemos perfazer um perfil da circulação de produções sobre o Psicodrama no cenário brasileiro de periódicos acadêmicos, a saber: (1) intensificação das produções de 2011 até 2014, que sobrepõe numericamente os anos anteriores; (2) predominância de artigos teóricos sobre o Psicodrama; (3) hegemonia de discussões voltadas à área clínica; (4) concentração de publicações em um único periódico; (5) ampla difusão de filiações institucionais relacionadas aos autores que produzem conhecimento sobre o Psicodrama e expressão de alguns escritores de filiações estrangeiras no Brasil.

\section{CONSIDERAÇÕES FINAIS}

Este trabalho traça um panorama das produções de artigos sobre o Psicodrama em periódicos nacionais, oferecendo uma análise de dados coletados com o esteio metodológico da revisão sistemática de literatura. A importância dessa proposta de pesquisa consiste em mapear as incursões que o Psicodrama tem feito nos meios científicos, a fim de proporcionar dados que sirvam de base para outras pesquisas e futuras ponderações sobre $\mathrm{o}$ desenvolvimento dessa abordagem no Brasil. O Psicodrama, segundo ajuizamos, está inserido no meio acadêmico e mantém ativo a herança de Moreno (MARINEAU, 2013).

A despeito disso, ressaltamos que os dados examinados devem ser lidos com cautela, por apresentarem somente uma leitura sobre a produção do conhecimento psicodramático no Brasil. Outras fontes de pesquisa que, por exemplo, poderiam aquilatar futuras possibilidades de entendimento sobre isso seriam revisões sistemáticas sobre teses e dissertações disponíveis em outras bases de dados e registro de produções vinculadas a eventos acadêmicos. Eis algumas sugestões para pesquisas que somariam contribuições ao apontamento de Gulassa e Kaufman (2014), apresentado no início deste trabalho.

Cumpre concluir que o incessante trabalho de revisão sistemática de produções acadêmicas é um processo que deve ser refeito com periodicidade e suplementado com outras pesquisas. Intencionamos que a tradição de fazer ciência e conhecer o que se produz dela no Psicodrama se amplie no Brasil.

\section{REFERÊNCIAS}

AKOBENG, A. Understanding systematic reviews and meta-analysis. Archives of Disease in Childhood. London, n. 90, p. 845-848, 2005.

GULASSA, D; KAUFMAN, A. Psicodrama no Século XXI: Integrar ou Morrer. Revista Brasileira de Psicodrama. São Paulo, v. 22, n. 1, p. 129-130, 2014.

LIBERALI, R; GROSSEMAN, S. Use of psychodrama in medicine in Brazil: a review of 
Branco, Paulo Coelho Castelo; Farias, Heitor Blesa; Carpes, Cândida de Oliveira; Leite, Laryssa Soares. Produção de artigos em Psicodrama no Brasil: revisão sistemática (1996-2014)

the literature. Interface. Botucatu, v. 19, n. 54, p. 561-571, 2015.

MARINEAU, R. Jacob Levy Moreno, 1889-1974: pai do psicodrama, da sociometria e da psicoterapia de grupo. São Paulo: Ágora, 1992.

A integração da herança de Moreno. Revista Brasileira de Psicodrama. São Paulo, v. 21, n. 1, p. 113-125, 2013.

ROJAS-BERMÚDEZ, J. Introdução ao psicodrama. São Paulo: Editora Mestre Jou, 1977.

Recebido: 16/12/2015

Aceito: $22 / 03 / 2016$

\section{Paulo Coelho Castelo Branco}

Docente do Curso de Psicologia da Universidade Federal da Bahia (UFBA) - Campus Anísio Teixeira. Doutor em Psicologia pela Universidade Federal de Minas Gerais (UFMG). Coordenador do Núcleo de Estudos em Psicologia Humanista (NEPH). Rua Rio de Contas, 58, Candeias, CEP: 45.029-094. Vitória da Conquista - BA. Tel.: (77) 3429 2730. E-mail: pauloccbranco@gmail.com

\section{Heitor Blesa Farias}

Graduando em Psicologia pela Universidade Federal da Bahia (UFBA) - Campus Anísio Teixeira. Bolsista PIBIC/FAPESB. Rua Rio de Contas, 58, Candeias, CEP: 45.029-094. Vitória da Conquista - BA. Tel.: (77) 3429 2730. E-mail: h_heitor@hotmail.com

\section{Cândida de Oliveira Carpes}

Graduanda em Psicologia pela Universidade Federal da Bahia (UFBA) - Campus Anísio Teixeira. Bolsista PIBIC/CNPq. Rua Rio de Contas, 58, Candeias, CEP: 45.029-094. Vitória da Conquista - BA. Tel.: (77) 3429 2730. E-mail: cândida.carpes@hotmail.com

\section{Laryssa Soares Leite}

Graduanda em Psicologia pela Universidade Federal da Bahia (UFBA) - Campus Anísio Teixeira. Bolsista PIBIC/UFBA. Rua Rio de Contas, 58, Candeias, CEP: 45.029-094. Vitória da Conquista - BA. Tel.: (77) 3429 2730. E-mail: 1aryssaasoares245@gmail.com 